\title{
Plasma Metabolomics Characteristic of Gout Patients With Regular Glucose, Blood Pressure, and Blood Lipid
}

\author{
Baoyu Zhang \\ Beijing Lu He Hospital, Capital Medical University \\ Lijie Zhang \\ Beijing Lu He Hospital, Capital Medical University \\ Yuan Wang \\ Beijing Lu He Hospital, Capital Medical University \\ Zongwei Wang \\ Beijing Lu He Hospital, Capital Medical University \\ Caiyan Mo \\ Beijing Lu He Hospital, Capital Medical University \\ Bin $\mathrm{Cao}$ \\ Beijing Lu He Hospital, Capital Medical University \\ Ruili Yin \\ Beijing Lu He Hospital, Capital Medical University \\ Jing Ke \\ Beijing Lu He Hospital, Capital Medical University \\ Longyan Yang ( $\sim$ lyyang15@ccmu.edu.cn ) \\ Beijing Lu He Hospital, Capital Medical University \\ Dong Zhao \\ Beijing Lu He Hospital, Capital Medical University
}

Research article

Keywords: Gout, Metabolomics, Citrate cycle, Cysteine and methionine metabolism, Glutamate metabolism

Posted Date: December 15th, 2020

DOI: https://doi.org/10.21203/rs.3.rs-126057/v1

License: (c) (7) This work is licensed under a Creative Commons Attribution 4.0 International License. Read Full License 


\section{Abstract}

Background

Gout is a metabolic disease and is the most common form of inflammatory arthritis affecting men. However, the pathogenesis of gout is still uncertain, and novel biomarkers are needed for early prediction and diagnosis of gout. The aim of this study is to reveal the metabolic alterations in plasma of gout patients and discover novel molecular biomarkers for early diagnosis.

Methods

Metabonomics was employed to screen and identify novel biomarkers of gout based on human plasma. Ultra High-Pressure Liquid Chromatography-Mass Spectrometry (UHPLC-MS) and orthogonal signal correction partial least squares discriminate analysis (OPLS-DA) were also used for metabonomics study. Kyoto Encyclopedia of Genes and Genomes (KEGG) and MetaboAnalyst were used for pathway enrichment analysis.

Results

In this study, 51 metabolites in positive ion mode and 39 metabolites in negative ion mode were selected as remarkable significant variables between gout and healthy control group. Four unique pathways were found, namely Citrate cycle (TCA cycle), cysteine and methionine metabolism and alanine, aspartate, and

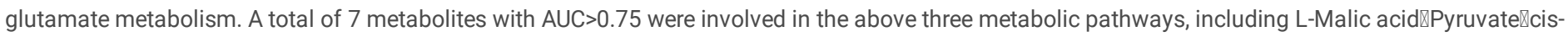
Aconitate, Cysteine-S-sulfate, L-2-Aminobutyric acid, L-Methionine, Succinic semialdehyde.

Conclusion

The present study identified the plasma metabolomics characteristic of gout through UHPLC-MS. The differential metabolites pathways of gout screened out were involved in Citrate cycle (TCA cycle), cysteine and methionine metabolism and alanine, aspartate, and glutamate metabolism. The metabolomics characteristic of gout could provide novel insights into the pathogenesis of gout.

\section{Background}

Gout is inflammatory arthritis caused by the deposition of monosodium urate(MSU) crystals in the joints[1]. Its prevalence has risen from $0.1 \%$ to about $10 \%$ globally, with 0.3 to 6 new gout patients per 1,000 people per year[2]. Without regular treatment, unresolved chronic inflammation and tissue remodeling can result in the formation of nodules called tophi, consisting of inflammatory granulomatous tissue and deposited MSU crystals, which can lead to joint damage and dysfunction. The recurrent gouty arthritis seriously affects the patient's life quality. The main pathological feature of gout is that chronic MSU crystals deposit in the joints and ligaments in the case of elevated serum uric acid[3]. Serum urate level is a strong nonlinear concentration-dependent predictor of acute gout onset, but the uric acid level does not predict the risk of gout. So far, the pathogenesis of gout is not fully understood.

More and more evidence shows that gout is a chronic metabolic disease caused by a combination of genetic, environmental, and lifestyle factors. Understanding the pathogenesis of gout requires a method that can reflect the combined effects of genetics, environment, and lifestyle factors. Metabolomics is a high-throughput technology that captures an individual's systemic metabolic state by simultaneously analyzing a wide range of low-molecular-weight compounds (metabolites) in a biological sample. Metabolomics can be used to discover new biomarkers by detecting and analyzing the changes of metabolites such as blood and urine. It is possible to have a new understanding of the biochemical changes accompanying the occurrence and development of diseases, and then to discover biomarkers and the molecular mechanism of pathology, finally achieves the purpose of guiding clinical diagnosis.

Recently, several studies have reported the preliminary application of metabolomics in gout. Most reviews were based on Gas Chromatography-Mass Spectrometer (GC-MS) or Nuclear Magnetic Resonance (NMR) spectroscopy platforms[4-7]. Very few studies have applied UHPLC-MS[8]. Existing studies have not excluded patients with other metabolic diseases such as hyperlipidemia, hypertension, and diabetes. In the present study, we utilized a UHPLC-MS to perform metabolomics analyses. We compared the metabolic products of the age- and gender-matched gout group and the healthy control group. All participants had regular glucose, blood pressure, and blood lipid.

\section{Methods}

\section{Study Design and Population}

This study was an across-sectional observational study. From October 2018 to May 2019, 30 participants and 30 volunteers were enrolled from the outpatient department or hospitalized at Center for Endocrine Metabolism and Immune Diseases, Beijing Luhe Hospital, Capital Medical University. Inclusion criteria for

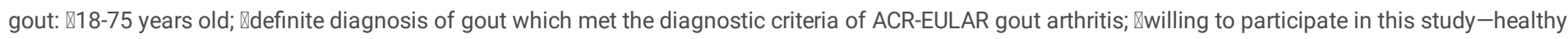
control group: Normal blood uric acid levels. Exclusion criteria: malignant tumors/hematological tumors; pregnant/lactating women or women planning to

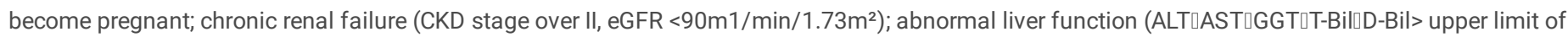
average value); hyperlipidemia (TG>1.7mmol/l, $\mathrm{CHO}>5.17 \mathrm{mmol} / \mathrm{I}, \mathrm{LDL}>3.36 \mathrm{mmol} / \mathrm{I})$; diabetes, hypertension, cardiovascular and cerebrovascular diseases; rheumatic diseases such as rheumatoid arthritis, osteoarthritis, spondyloarthropathy, etc.; have taken the following drugs in the last 6 months: glucocorticoids, immunosuppressants (mercaptopurine, 6-mercaptopurine, cyclosporine, cyclophosphamide), chemotherapy drugs; those who are allergic to drugs such as non-steroidal anti-inflammatory drugs, colchicine, febuxostat, allopurinol, or hypersensitivity; those with severe infections, active tuberculosis; have accepted urate-lowering therapy in the past three months; persons with mental illness who cannot cooperate; combined with other diseases affecting the efficacy or poor compliance. 
The healthy control group was age-matched healthy volunteers registered in the physical examination center of Beijing Luhe Hospital. This study was approved by the ethics committee of the Beijing Luhe Hospital, Capital Medical University. Written informed consent was obtained from each subject.

\section{Sample collection and preparation}

Venous plasma samples were collected in K2 EDTA vacutainer tubes from gout patients and HCs after an overnight fast for at least eight hours and cooled down in freezer $\left(4^{\circ} \mathrm{C}\right)$ at once. They were then centrifuged at $3000 \mathrm{rpm}$ for $10 \mathrm{~min}$ at $4^{\circ} \mathrm{C}$ within $2 \mathrm{~h}$. Supernatants (plasma) were separated and transferred into new vials, and immediately stored frozen $\left(-80^{\circ} \mathrm{C}\right)$ until sample preparation.

Before LC-MS analysis, $100 \mu \mathrm{L}$ of the sample was transferred to an EP tube, and $400 \mu \mathrm{L}$ extract solution (acetonitrile: methanol $=1: 1$ ) containing internal standard (L-2-Chlorophenylalanine, $2 \mu \mathrm{g} / \mathrm{mL}$ ) was added. After $30 \mathrm{~s}$ vortex, the samples were sonicated for $10 \mathrm{~min}$ in an ice-water bath. Then the samples were incubated at $-40^{\circ} \mathrm{C}$ for $1 \mathrm{~h}$ and centrifuged at $10000 \mathrm{rpm}$ for $15 \mathrm{~min}$ at $4^{\circ} \mathrm{C} .425 \mu \mathrm{L}$ of supernatant was transferred to a fresh tube and dried in a vacuum concentrator at $37^{\circ} \mathrm{C}$. Then, the dried samples were reconstituted in $200 \mu \mathrm{L}$ of $50 \%$ acetonitrile by sonication on ice for $10 \mathrm{~min}$. The constitution was then centrifuged at $13000 \mathrm{rpm}$ for $15 \mathrm{~min}$ at $4^{\circ} \mathrm{C}$, and $75 \mu \mathrm{L}$ of supernatant was transferred to a fresh glass vial for $\mathrm{LC} / \mathrm{MS}$ analysis. The quality control (QC) sample was prepared by mixing an equal aliquot of the supernatants from all of the samples.

\section{UHPLC-MS/MS metabolomics profiling}

The UHPLC separation was carried out using a 1290 Infinity series UHPLC System (Agilent Technologies), equipped with a UPLC BEH Amide column (2.1 *

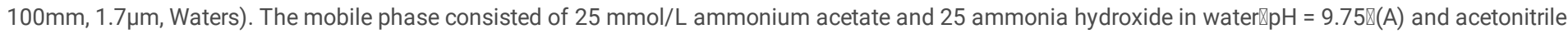
(B). The analysis was carried with elution gradient as follows: 0 0.5min, 95\% B; 0.5 7.0min, 95\% 65\% B; 7.0 8.0min, 65\% 40\% B; 8.0 9.0min, 40\% B; 9.0 9.1 min, 40\% 95\% B; 9.1 12.0min, 95\% B. The column temperature was $25^{\circ} \mathrm{C}$. The auto-sampler temperature was $4^{\circ} \mathrm{C}$, and the injection volume was $1 \mu \mathrm{L}$ (pos) or $1 \mu \mathrm{L}$ (neg), respectively.

The Triple TOF 6600 mass spectrometry (AB Sciex) was used for its ability to acquire MS/MS spectra on an information-dependent basis (IDA) during an LC/MS experiment. In this mode, the acquisition software (Analyst TF 1.7, AB Sciex) continuously evaluates the full scan survey MS data as it collects and triggers the acquisition of MS/MS spectra depending on preselected criteria. In each cycle, the most intensive 12 precursor ions with intensity above 100 were chosen for MS/MS at collision energy (CE) of $30 \mathrm{eV}$. The cycle time was $0.56 \mathrm{~s}$. ESI source conditions were set as follows: Gas 1 as 60 psi, Gas 2 as 60 psi, Curtain Gas as $35 \mathrm{psi}$, Source Temperature as $600^{\circ} \mathrm{C}$, Declustering potential as $60 \mathrm{~V}$, lon Spray Voltage Floating (ISVF) as $5000 \mathrm{~V}$ or -4000V in positive or negative modes, respectively.

\section{Data preprocessing and statistical analysis}

MS raw data (.wiff) files were converted to the mzXML format by ProteoWizard, and processed by R package XCMS (version 3.2). The process includes peak deconvolution, alignment, and integration. Minfrac and cut-off are set as 0.5 and 0.6 respectively. In-house MS2 database was applied for metabolites identification.

In this study, the original data were preprocessed firstly. Then, the missing values were filled up by half of the minimum value. Also, the internal standard normalization method was employed in this data analysis. The final dataset containing the information of peak number, sample name, and normalized peak area was imported to SIMCA15.0.2 software package (Sartorius Stedim Data Analytics AB, Umea, Sweden) for multivariate analysis. Data were scaled and logarithmically transformed to minimize the impact of both noise and high variance of the variables. After these transformations, PCA, an unsupervised analysis that reduces the dimension of the data, was carried out to visualize the distribution and the grouping of the samples. $95 \%$ confidence interval in the PCA score plot was used as the threshold to identify potential outliers in the dataset. To visualize group separation, and find significantly changed metabolites, supervised orthogonal projections to OPLS-DA was applied. Then, 7-fold cross-validation was performed to calculate the value of R2 and Q2. R2 indicates how well the variation of a variable is explained, and Q2 means how well a variable could be predicted. To check the robustness and predictive ability of the OPLS-DA model, 200 times permutations were further conducted. Afterwards, the R2 and Q2 intercept values were obtained. Here, the intercept value of Q2 represents the robustness of the model, the risk of overfitting and the reliability of the model, which will be the smaller the better. Furthermore, the value of VIP of the first principal component in OPLS-DA analysis was obtained. It summarizes the contribution of each variable to the model. The metabolites with VIP $>1, p<0.05$ (student t-test) and fold change $>1.2$ or $<0.83$ were considered as significantly changed metabolites. Besides, commercial databases, including KEGG (http://www.genome.jp/kegg/) and MetaboAnalyst (http://www.metaboanalyst.ca/) were used for pathway enrichment analysis.

\section{Results}

\section{Demographic characteristics of the study participants}

In this study, 30 male gout patients $₫ 30$ age, gender, and body mass index (BMI)-matched healthy controls (HCs) were included. The average age of the participants was $33.78 \pm 9.05$ years, and the mean BMI was $25.50 \pm 2.90 \mathrm{~kg} / \mathrm{m} 2$. Gout patients had higher blood pressure(BP), uric acid (UA), glucose (GLU), triglycerides (TG), total cholesterol (TC), low-density lipoprotein cholesterol (LDL-C), creatinine (Cr), and blood urea nitrogen (BUN) compared with healthy controls. Demographic characteristics of gout patients and healthy controls were shown in Table 1.

\section{Identification of plasma differential metabolites}

A total of 1505 features in positive ion mode and 1608 features in negative ion mode were detected using HUPLC-MS. After relative standard deviation denoising and filling up the missing values by half of the minimum value, 1492 features in positive ion mode and 1589 features in negative ion mode remained. 
The remained data were imported into a SIMCA software package to conduct multivariate statistical analysis. The principal component analysis (PCA) analysis could outline the original distribution of metabolites. As shown in Fig.1a and b, there were no obvious outlier samples in modes. However, the scatter plot failed to show a clear separation in modes. Then, the OPLS-DA model was used to characterize the metabolic disturbances (Figure1.c and d). The cumulative values of $R 2 Y(R 2 Y=0.731)$ and $Q 2 Y(Q 2 Y=0.593)$ in positive ion mode and $R 2 Y(R 2 Y=0.891)$ and $Q 2 Y(Q 2 Y=0.739)$ in negative ion mode indicated that the models had an excellent predictive and interpretative capacity. Gout samples could be clearly distinguished from healthy control samples of positives and negative ion modes. Permutation plots of two OPLS-DA models repeated 200 times verified the validation of the models (Fig. $1 \mathrm{e}$ and $\mathrm{f}$ ). All the permuted R2 (cum)and Q2 (cum) values to the left were lower than the original point to the right, and all the regression lines of the Q2 (cum) points have a negative intercept $[9,10]$, which indicates that the models were valid with no overfitting.

The standard value used in this project was that the P-value $<0.05$, and Variable Importance in the Projection (VIP) value $>1.0,80$ and 62 metabolites were selected as remarkable significant variables in the two modes between gout and control group. According to fold change $>1.2$ or $<0.83,56$ and 45 potential metabolic biomarkers were remained in positive and negative ion mode, respectively. Among the potential metabolic biomarkers, 51 metabolites positive ion mode and 39 metabolites negative ion mode can find identifier (ID) in the Human Metabolome Database (HMDB) (Figure.2a,2b). Correlation analysis of metabolite differential was shown in Figure.2c,2d. The differential metabolites can be categorized as amino acids, purine derivatives, fatty acyls, carboxylic acids and derivatives. ROC analysis of each metabolite was conducted to seek novel diagnostic biomarkers. Among 90 metabolites, $40 \%$ presented high AUC values (AUC >0.75). The HMDB ID, VIP, P-values, fold change, and AUC of each metabolite was shown in detail (Table2.). Using uric acid as a diagnostic indicator of gout, compared with healthy control, the AUC was 0.948 . If using thymine as a diagnostic indicator of gout, the AUC was 0.949 , and 5 metabolites(Thymine, N-Acetylcadaverine, Cyclohexylamine, Monomethyl glutaric acid, Val-Thr, N-Acetyl-L-alanine) AUC>0.9.

\section{Pathway analysis of gout}

To investigate whether metabolism is affected by gout, metabolites with an AUC area greater than 0.75 were selected for metabolic pathway enrichment analysis. We performed pathway analysis using MetaboAnalyst. The P-value and the pathway impact were calculated from the Metabolite Set Enrichment Analysis(MSEA) and the pathway topology analysis, respectively. The P-value threshold was set 0.05 , and the values above this threshold were filtered out as insignificant pathways. As a result, we found four unique pathways. They were TCA cycle, glyoxylate and dicarboxylate metabolism, cysteine and methionine metabolism and alanine, aspartate and glutamate metabolism (Figure 3). Glyoxylate and dicarboxylate metabolism occur in plants and microorganisms. It has the same metabolites as TCA cycle. So there were three metabolic pathways identified in our study. A total of 7 metabolites with AUC>0.75 were involved in the above three metabolic pathways, including L-Malic acid『Pyruvate『cis-Aconitate, Cysteine-S-sulfate, L-2-Aminobutyric acid, L-Methionine, Succinic semialdehyde(Figure 4, Figure5).

\section{Correlation analysis of clinical features and metabolites}

The correlation analysis between metabolites and clinical indicators showed that L-Malic acid囚Pyruvate『cis-Aconitate, L-2-Aminobutyric acid, L-Methionine were correlated negatively with blood pressure, glucose, uric acid, and blood lipid. Succinic semialdehyde and cysteine-S-sulfate were correlated positively with blood pressure, glucose, uric acid, and blood lipid(Figure 6).

\section{Discussion}

we enrolled the patients with gout and healthy control group, compared to the difference of plasma metabolites. Unlike previous studies, this study excluded obesity, hyperlipidemia, hypertension, which could be better reflect the difference metabolites between gout and healthy control. We still observed that blood glucose and systolic blood pressure were higher in the gout group than in the healthy controls, which indicated that uric acid was related to glucose and salt metabolism[11-14].

In this study, 51 metabolites in positive ion mode and 39 metabolites in negative ion mode were identified as different metabolites that involved amino acid metabolism, purine metabolism, carbohydrate metabolism, and fatty metabolism. However, many new features were still present, but there were no matches in the database of known metabolites. The identification of these unknown substances using HPLC-MS will be focused on in our future work. HPLC-MS -based metabolomics is a promising technique for biomarker discovery.

We found that uric acid was a perfect biomarker for the diagnosis of gout. However, using thymine as a diagnostic indicator of gout, the AUC was 0.949 , which was better than uric acid. These bases are the building blocks of DNA and life forms on earth. We found five metabolites (N-Acetylcadaverine,

Cyclohexylamine, Monomethyl glutaric acid, Val-Thr, N-Acetyl-L-alanine) which AUC>0.9. However, no metabolic pathways, including the five metabolites, were found in the analysis of metabolic pathways. The role of these five metabolites needs further study.

In the enrichment analysis of metabolic pathways, we found three specific pathways. The TCA cycle is an important aerobic pathway for the final steps of the oxidation of carbohydrates. As an intermediate metabolite of the TCA cycle, the content of L-Malic acid, pyruvate, and Cis-aconitate was significantly lower in the gout group than in the healthy control group in our study. L-malic acid reduces oxidative stress and increases the mitochondrial antioxidative defenses. In

Zeng's study, L-malate significantly reduces reactive oxygen species (ROS) and malondialdehyde content levels in the liver and heart of aged rats[15]. L-malate might increase the antioxidant capacity of mitochondria by enhancing the expression of mRNAs involved in the malate synthase and the antioxidant enzymes[15]. Pyruvate is the end-product of glycolysis, is derived from additional sources in the cellular cytoplasm, and is ultimately destined for transport into mitochondria as a master fuel input undergirding citric acid cycle carbon flux. In mitochondria, Pyruvate drives ATP production by oxidative phosphorelation and multiple biosynthetic pathways intersecting the citric acid cycle. Appropriate regulation of pyruvate flux is critical for maintaining cellular function in multiple contexts. 
Pyruvate dysmetabolism presents in chronic, progressive diseases such as chronic obstructive pulmonary disease (COPD), obesity, diabetes, and aging[16]. The mitochondrial pyruvate carrier mediates high fat diet-induced increases in hepatic TCA cycle capacity[17]. In our study, L-Malic acid, pyruvate and Cisaconitate in the gout group were lower than those in the normal control group. It was speculated that the energy metabolism of the TCA cycle was slowed down, and mitochondrial function was affected in patients with gout. Studies have reported that excess uric acid induced oxidative stress, triglyceride accumulation, and mitochondrial dysfunction in the liver and hyperuricemia induced mitochondrial dysfunction and triglyceride accumulation in skeletal muscle. These findings may explain why hyperuricemia is an independent predictor of diabetes[18].

In our study, in the cysteine and methionine metabolism pathway, the cysteine-S-sulfate level in the gout group was elevated. In contrast, the L-methionine and L-2-aminobutyric acid level were lower than those in the control group. Methionine is an essential amino acid in humans, as the substrate for other amino acids such as cysteine and taurine, versatile compounds such as S-Adenosyl methionine, and the critical antioxidant glutathione. Cysteine-S-sulfate is an abnormal metabolite discovered in the urine and blood of a patient with cysteine oxidase deficiency, a rare disorder of sulfur amino acid metabolism associated with brain damage and mental retardation. L-methionine is levorotatory of methionine. Methionine promotes phospholipids methylation in the liver cell membrane, boots the membrane fluidity and strengthens the $\mathrm{Na}^{+}-\mathrm{K}^{+}$ATPase pump function. Methionine reduces bile aggradations of the liver cells, and has a protective effect on the liver. Thus it enhances the cysteine, glutathione, and taurine synthesis, reduces the bile acid accumulation in the liver, and strengthens detoxification. Lack of methionine can lead to a blockage in protein synthesis in the body, causing damage to the body. Methionine- and cholinedeficient diets (MCDD) induce fatty liver in mice which are partly mediated by TG lipolysis in white adipose tissues (WATs)[19]. The plasma concentration of Lmethionine was reduced in the gout group, which may be associated with a predisposition to fatty liver in patients with gout. In a 6-year study, the author found that the methionine to homocysteine ratio was associated with dementia development and structural brain. It was suggesting that a higher methionine to homocysteine ratio may be important in reducing the rate of brain atrophy and decreasing the risk of dementia in older adults[20]. L-2-aminobutyric acid is a kind of non-natural chiral amino acid, which has the functions of inhibiting human nerve information transmission, enhancing the activity of glucose phosphatase and promoting the metabolism of brain cells. A study demonstrated that oral administration of L-2-aminobutyric acid -AB efficiently raised both circulating and myocardial glutathione levels and protected against doxorubicin-induced cardiomyopathy in mice[21].

In the present study, we found the plasma level of succinic semialdehyde evaluated and pyruvate reduced. The role of gout metabolism and succinic semialdehyde in gout metabolism is unclear and needs further research.

Our study was a cross-sectional observational study, which found that some metabolites might be used in the diagnosis of gout or hyperuricemia. However, the causal relationship cannot be determined. To better understand the physiological and pathological mechanisms of gout and hyperuricemia, the mechanism of the role of metabolites in the pathogenesis of gout needs further verification.

\section{Conclusions}

In summary, the present study identified the plasma metabolomics signatures of gout through LC-MS. The differential metabolites of gout screened out were involved in the TCA cycle, cysteine and methionine metabolism and alanine, aspartate and glutamate metabolism. These results can provide novel insights into the development of gout.

\section{Abbreviations}

Ultra High-Pressure Liquid Chromatography-Mass Spectrometry (UHPLC-MS); Orthogonal signal correction partial least squares discriminate analysis (OPLSDA); Hyperuricemia (HUA); Area under the curve (AUC); Gas Chromatography-Mass Spectrometer (GC-MS); Nuclear magnetic resonance (NMR); Body mass index (BMI); Healthy controls (HCs); Systolic blood pressure (SBP); Uric acid (UA); Glucose (GLU); Albumin (ALB); Principal component analysis (PCA); Variable Importance in the Projection (VIP); Human Metabolome Database (HMDB); International Union of Pure and Applied Chemistry (IUPAC); Adenosine triphosphate (ATP); Adenosine diphosphate (ADP); Quality control (QC); Information-dependent basis (IDA); collision energy (CE); lon Spray Voltage Floating (ISVF); Diastolic blood pressure (DBP); white blood cell (WBC); Hemoglobin (HGB); platelet (PLT); Total protein (TP); Alanine aminotransferase (ALT); Aspartate aminotransferase (AST); -glutamyl transpeptidase ( $\mathrm{Y}-\mathrm{GGT})$; Blood urea nitrogen (BUN); Calcium (Ca); Phosphorus (P); lactate dehydrogenase (LDH); Triglycerides (TG); Total cholesterol (TC); High-density lipoprotein (HDL-C); Low-density lipoprotein cholesterol (LDL-C); Fold change (FC).

\section{Declarations}

\section{Acknowledgements}

Not applicable.

\section{Authors' contributions}

ZBY and ZLJ designed the project and supervised its implementation. WY, WZW, and MCY enrolled and collected blood samples. CB and YRL analyzed statistical data. KJ was in charge of quality control. YLY managed the database and specimen library. ZD directed the project. ZBY and MCY writed the manuscript. All authors read and approved the final manuscript.

\section{Funding}

Not applicable.

\section{Availability of data and materials}


All data obtained or analyzed in this study are included in this manuscript and its supplementary information file.

\section{Ethics approval and consent to participate}

Informed consent was obtained from all subjects following the Declaration of Helsinki. The Institutional Review Board of Beijing Luhe Hospital approved all research on human subjects.

\section{Consent for publication}

All patients provided written informed consent for the publication of information resulting from the results without any personally identifying information.

\section{Competing interests}

The authors declare that they have no competing interests.

\section{Author Details}

${ }^{1}$ Beijing Key Laboratory of Diabetes Research and Care, Center for Endocrine Metabolism and Immune Diseases, Lu He Hospital, Capital Medical University, Beijing, China.

\section{References}

1. Dalbeth N, Merriman TR, Stamp LK: Gout. Lancet 2016, 388(10055):2039-2052.

2. Pascart T, Liote F: Gout: state of the art after a decade of developments. Rheumatology (Oxford) 2019, 58(1):27-44.

3. McCarty DJ, Hollander JL: Identification of urate crystals in gouty synovial fluid. Ann Intern Med 1961, 54:452-460.

4. Luo Y, Wang L, Peng A, Liu JY: Metabolic profiling of human plasma reveals the activation of 5-lipoxygenase in the acute attack of gouty arthritis. Rheumatology (Oxford) 2019, 58(2):345-351.

5. Li Q, Wei S, Wu D, Wen C, Zhou J: Urinary Metabolomics Study of Patients with Gout Using Gas Chromatography-Mass Spectrometry. Biomed Res Int 2018, 2018:3461572.

6. Luo Y, Wang L, Liu XY, Chen X, Song YX, Li XH, Jiang C, Peng A, Liu JY: Plasma profiling of amino acids distinguishes acute gout from asymptomatic hyperuricemia. Amino Acids 2018, 50(11):1539-1548.

7. Zhang Y, Zhang H, Chang D, Guo F, Pan H, Yang Y: Metabolomics approach by (1)H NMR spectroscopy of serum reveals progression axes for asymptomatic hyperuricemia and gout. Arthritis Res Ther 2018, 20(1):111.

8. Huang Y, Xiao M, Ou J, Lv Q, Wei Q, Chen Z, Wu J, Tu L, Jiang Y, Zhang X et al: Identification of the urine and serum metabolomics signature of gout. Rheumatology (Oxford) 2020, 59(10):2960-2969.

9. Pasikanti KK, Esuvaranathan K, Ho PC, Mahendran R, Kamaraj R, Wu QH, Chiong E, Chan EC: Noninvasive urinary metabonomic diagnosis of human bladder cancer. J Proteome Res 2010, 9(6):2988-2995.

10. Mahadevan S, Shah SL, Marrie TJ, Slupsky CM: Analysis of metabolomic data using support vector machines. Anal Chem 2008, 80(19):7562-7570.

11. Krishnan E, Akhras KS, Sharma H, Marynchenko M, Wu EQ, Tawk R, Liu J, Shi L: Relative and attributable diabetes risk associated with hyperuricemia in US veterans with gout. QJM 2013, 106(8):721-729.

12. Bhole V, Choi JW, Kim SW, de Vera M, Choi H: Serum uric acid levels and the risk of type 2 diabetes: a prospective study. Am J Med 2010, 123(10):957961.

13. Juraschek SP, McAdams-Demarco M, Miller ER, Gelber AC, Maynard JW, Pankow JS, Young H, Coresh J, Selvin E: Temporal relationship between uric acid concentration and risk of diabetes in a community-based study population. Am J Epidemiol 2014, 179(6):684-691.

14. Liu J, Tao L, Zhao Z, Mu Y, Zou D, Zhang J, Guo X: Two-Year Changes in Hyperuricemia and Risk of Diabetes: A Five-Year Prospective Cohort Study. J Diabetes Res 2018, 2018:6905720.

15. Zeng X, Wu J, Wu Q, Zhang J: L-malate enhances the gene expression of carried proteins and antioxidant enzymes in liver of aged rats. Physiol Res 2015 , 64(1):71-78.

16. Gray LR, Tompkins SC, Taylor EB: Regulation of pyruvate metabolism and human disease. Cell Mol Life Sci 2014, 71(14):2577-2604.

17. Rauckhorst AJ, Gray LR, Sheldon RD, Fu X, Pewa AD, Feddersen CR, Dupuy AJ, Gibson-Corley KN, Cox JE, Burgess SC et al: The mitochondrial pyruvate carrier mediates high fat diet-induced increases in hepatic TCA cycle capacity. Mol Metab 2017, 6(11):1468-1479.

18. Maarman GJ, Andrew BM, Blackhurst DM, Ojuka EO: Melatonin protects against uric acid-induced mitochondrial dysfunction, oxidative stress, and triglyceride accumulation in C2C12 myotubes. J Appl Physiol (1985) 2017, 122(4):1003-1010.

19. Cheng Y, Gan-Schreier H, Seessle J, Staffer S, Tuma-Kellner S, Khnykin D, Stremmel W, Merle U, Herrmann T, Chamulitrat W: Methionine- and CholineDeficient Diet Enhances Adipose Lipolysis and Leptin Release in aP2-Cre Fatp4-Knockout Mice. Mol Nutr Food Res 2020, 64(22):e2000361.

20. Hooshmand B, Refsum H, Smith AD, Kalpouzos G, Mangialasche F, von Arnim CAF, Kareholt I, Kivipelto M, Fratiglioni L: Association of Methionine to Homocysteine Status With Brain Magnetic Resonance Imaging Measures and Risk of Dementia. JAMA Psychiatry 2019, 76(11):1198-1205.

21. Irino Y, Toh R, Nagao M, Mori T, Honjo T, Shinohara M, Tsuda S, Nakajima H, Satomi-Kobayashi S, Shinke T et al: 2-Aminobutyric acid modulates glutathione homeostasis in the myocardium. Sci Rep 2016, 6:36749. 
Tables

Table.1 Demographic characteristic of gout patients, hyperuricemia patients and healthy controls

\begin{tabular}{|c|c|c|c|c|}
\hline & $\mathrm{HCs} \otimes n=30 \bigotimes$ & Gout $\rrbracket n=30 \bigotimes$ & $\mathrm{W} / \mathrm{F} / \mathrm{t}$ & $P$ \\
\hline Age $(m e a n \pm S D)$ & $33.90 \pm 8.09$ & $32.97 \pm 9.20$ & 491.50 & 0.544 \\
\hline $\mathrm{BMI}($ mean $\pm S D)$ & $25.24 \pm 3.21$ & $25.81 \pm 2.63$ & -0.74 & 0.460 \\
\hline $\mathrm{SBP}(\mathrm{mmHg})$ & $114.73 \pm 16.34$ & $120.33 \pm 7.90$ & 275.00 & $0.009 *$ \\
\hline $\mathrm{DBP}(\mathrm{mmHg})$ & $73.23 \pm 8.47$ & $80.70 \pm 6.52$ & 276.50 & $0.007^{\star}$ \\
\hline WBC $\left(10^{\wedge} 9 / \mathrm{L}\right)$ & $6.67 \pm 1.58$ & $7.73 \pm 1.81$ & 243.00 & $0.026^{\star}$ \\
\hline $\operatorname{RBC}\left(10^{\wedge} 12 / L\right)$ & $5.10 \pm 0.38$ & $5.07 \pm 0.37$ & 365.00 & 0.872 \\
\hline $\mathrm{HGB}(\mathrm{g} / \mathrm{L})$ & $153.87 \pm 11.06$ & $152.44 \pm 10.40$ & 0.489 & 0.627 \\
\hline $\operatorname{PLT}\left(10^{\wedge} 9 / \mathrm{L}\right)$ & $243.20 \pm 53.07$ & $229.68 \pm 59.98$ & 430.00 & 0.357 \\
\hline $\mathrm{TP}(\mathrm{g} / \mathrm{L})$ & $73.67 \pm 3.92$ & $75.71 \pm 4.02$ & 337.5 & 0.098 \\
\hline $\operatorname{ALB}(\mathrm{g} / \mathrm{L})$ & $47.08 \pm 2.34$ & $49.45 \pm 2.43$ & -3.84 & $<0.001$ * \\
\hline $\operatorname{ALT}(\mathrm{U} / \mathrm{L})$ & $17.30 \pm 6.25$ & $23.07 \pm 11.62$ & 323.00 & 0.061 \\
\hline $\mathrm{AST}(\mathrm{U} / \mathrm{L})$ & $18.17 \pm 4.04$ & $20.21 \pm 9.95$ & 440.50 & 0.894 \\
\hline$y-G G T(U / L)$ & $22.73 \pm 8.16$ & $32.83 \pm 24.92$ & 357.50 & 0.1734 \\
\hline $\mathrm{GLU}(\mathrm{mmol} / \mathrm{L})$ & $4.88 \pm 0.39$ & $5.25 \pm 0.42$ & -3.52 & $0.001^{*}$ \\
\hline $\mathrm{Cr}($ umol/L) & $81.32 \pm 9.07$ & $87.33 \pm 12.13$ & -2.13 & $0.038 *$ \\
\hline $\mathrm{BUN}(\mathrm{mmol} / \mathrm{L})$ & $4.92 \pm 1.07$ & $4.23 \pm 1.11$ & 612.50 & $0.017 *$ \\
\hline $\mathrm{Ca}(\mathrm{mmol} / \mathrm{L})$ & $2.39 \pm 0.11$ & $2.41 \pm 0.08$ & -0.69 & 0.490 \\
\hline $\mathrm{P}(\mathrm{mmol} / \mathrm{L})$ & $1.04 \pm 0.16$ & $1.13 \pm 0.13$ & -2.28 & $0.026^{\star}$ \\
\hline UA(umol/L) & $348.13 \pm 50.53$ & $503.03 \pm 82.46$ & 47.00 & $<0.001$ * \\
\hline $\mathrm{TG}(\mathrm{mmol} / \mathrm{L})$ & $0.89 \pm 0.29$ & $1.10 \pm 0.34$ & 286.00 & $0.016^{*}$ \\
\hline $\mathrm{TC}(\mathrm{mmol} / \mathrm{L})$ & $4.20 \pm 0.57$ & $4.50 \pm 0.60$ & -2.04 & 0.046 \\
\hline $\mathrm{HDL}-\mathrm{C}(\mathrm{mmol} / \mathrm{L})$ & $1.30 \pm 0.19$ & $1.22 \pm 0.23$ & 1.42 & 0.161 \\
\hline LDH-C(mmol/L) & $2.56 \pm 0.52$ & $2.90 \pm 0.52$ & -2.58 & $0.012^{\star}$ \\
\hline
\end{tabular}

HCs: healthy controls; SBP: systolic blood pressure; DBP: diastolic blood pressure; BMI: body mass index; WBC: white blood cell; HGB: haemoglobin; PLT: platelet; TP: total protein; ALB: albumin; ALT: alanine aminotransferase; AST: aspartate aminotransferase; $\gamma$-GGT:Y-glutamyl transpeptidase; GLU: glucose: Cr: creatinine; BUN: blood urea nitrogen; Ca: calcium; P: phosphorus; UA: Uric acid; TG: Triglycerides; TC: total cholesterol; HDL-C: high-density lipoprotein; LDL-C: low-density lipoprotein cholesterol;

* HCs vs Gout $p<0.05$

Table.2. The HMDB ID, VIP, P-values, fold change, and AUC of each metabolite 


\begin{tabular}{|c|c|c|c|c|c|c|c|c|c|c|c|c|c|}
\hline mode & MS2 name & $\mathrm{mz}$ & rt & $\begin{array}{l}\text { MEAN } \\
\text { Gout }\end{array}$ & $\begin{array}{l}\text { MEAN } \\
\text { Control }\end{array}$ & VIP & $\begin{array}{l}\text { P- } \\
\text { VALUE }\end{array}$ & $\begin{array}{l}\text { FOLD } \\
\text { CHANGE }\end{array}$ & HMDB & $\begin{array}{l}\text { ROC } \\
\text { AUC }\end{array}$ & lower & upper & st \\
\hline$N$ & L-Malic acid & 133.014 & 388.502 & 0.152 & 0.294 & 2.407 & 0.000 & 0.517 & HMDB00156 & 0.884 & 0.804 & 0.965 & $F_{c}$ \\
\hline $\mathrm{N}$ & Caprylic acid & 143.107 & 49.817 & 1.321 & 0.984 & 1.985 & 0.000 & 1.342 & HMDB00482 & 0.796 & 0.686 & 0.905 & $F_{c}$ \\
\hline$N$ & Pyruvate & 147.029 & 375.958 & 0.080 & 0.114 & 1.816 & 0.001 & 0.707 & HMDB00243 & 0.777 & 0.657 & 0.896 & $\mathrm{Al}$ \\
\hline$N$ & Cytosine & 170.056 & 184.777 & 0.042 & 0.033 & 1.919 & 0.001 & 1.298 & HMDB00630 & 0.760 & 0.637 & 0.883 & $P$ \\
\hline$N$ & cis-Aconitate & 173.009 & 421.385 & 0.320 & 0.396 & 1.679 & 0.000 & 0.807 & HMDB00072 & 0.763 & 0.644 & 0.883 & Ci \\
\hline$N$ & L-Ascorbic acid & 175.024 & 352.155 & 0.025 & 0.049 & 2.029 & 0.000 & 0.516 & HMDB00044 & 0.832 & 0.727 & 0.938 & $\mathrm{Fl}$ \\
\hline$N$ & Thymine & 185.056 & 287.034 & 0.068 & 0.139 & 2.667 & 0.000 & 0.492 & HMDB00262 & 0.949 & 0.895 & 1.000 & $\mathrm{Di}$ \\
\hline$N$ & D-galacturonic acid & 193.035 & 371.200 & 0.073 & 0.040 & 2.331 & 0.000 & 1.799 & HMDB02545 & 0.869 & 0.782 & 0.956 & P) \\
\hline$N$ & Cysteine-S-sulfate & 199.968 & 289.757 & 0.035 & 0.016 & 2.121 & 0.000 & 2.161 & HMDB00731 & 0.860 & 0.752 & 0.968 & $\mathrm{Ci}$ \\
\hline $\mathrm{N}$ & 3'-0-methyladenosine & 297.136 & 284.508 & 0.029 & 0.035 & 1.452 & 0.004 & 0.819 & HMDB06023 & 0.751 & 0.624 & 0.878 & $\mathrm{Pl}$ \\
\hline $\mathrm{N}$ & $\begin{array}{l}\text { Sphingosine-1- } \\
\text { phosphate }\end{array}$ & 378.238 & 263.835 & 0.072 & 0.098 & 2.222 & 0.000 & 0.735 & HMDB00277 & 0.864 & 0.774 & 0.955 & $\mathrm{pr}$ \\
\hline$P$ & $\mathrm{~N}$-Acetylcadaverine & 127.122 & 170.704 & 0.005 & 0.018 & 2.684 & 0.000 & 0.248 & HMDB02284 & 0.927 & 0.843 & 1.000 & $\mathrm{C}:$ \\
\hline$P$ & Dopamine & 136.075 & 284.744 & 0.144 & 0.174 & 1.975 & 0.000 & 0.828 & HMDB00073 & 0.782 & 0.667 & 0.898 & Bt \\
\hline$P$ & Hypoxanthine & 137.045 & 163.617 & 0.700 & 0.319 & 1.358 & 0.001 & 2.194 & HMDB00157 & 0.758 & 0.638 & 0.878 & $\mathrm{Pl}$ \\
\hline$P$ & Cyclohexylamine & 141.138 & 162.107 & 0.009 & 0.037 & 2.687 & 0.000 & 0.238 & HMDB31404 & 0.921 & 0.833 & 1.000 & OI \\
\hline$P$ & 1,2,3-Benzenetriol & 144.064 & 221.482 & 0.011 & 0.017 & 1.589 & 0.000 & 0.631 & HMDB13674 & 0.767 & 0.645 & 0.889 & be \\
\hline$P$ & L-2-Aminobutyric acid & 145.096 & 294.306 & 0.035 & 0.083 & 2.213 & 0.000 & 0.417 & HMDB00452 & 0.890 & 0.795 & 0.985 & ar \\
\hline$P$ & L-Methionine & 150.057 & 268.346 & 0.073 & 0.088 & 1.867 & 0.000 & 0.830 & HMDB00696 & 0.824 & 0.718 & 0.931 & $\mathrm{Ci}$ \\
\hline$P$ & $\begin{array}{l}\text { trans-2- } \\
\text { Hydroxycinnamic acid }\end{array}$ & 165.054 & 284.736 & 0.151 & 0.184 & 2.117 & 0.000 & 0.824 & HMDB02641 & 0.807 & 0.698 & 0.915 & $\begin{array}{l}\text { hy } \\
\text { ac }\end{array}$ \\
\hline$P$ & L-Pyroglutamic acid & 171.075 & 380.015 & 0.025 & 0.041 & 2.650 & 0.000 & 0.602 & HMDB00267 & 0.864 & 0.774 & 0.954 & Ci \\
\hline$P$ & L-Citrulline & 176.102 & 372.479 & 0.146 & 0.180 & 2.229 & 0.000 & 0.813 & HMDB00904 & 0.803 & 0.693 & 0.913 & $\mathrm{Ci}$ \\
\hline$P$ & Carbamazepine & 201.086 & 352.620 & 0.014 & 0.020 & 2.607 & 0.000 & 0.688 & HMDB14704 & 0.866 & 0.777 & 0.954 & di \\
\hline$P$ & Cysteine-S-sulfate & 201.982 & 291.722 & 0.011 & 0.004 & 2.302 & 0.000 & 2.364 & HMDB00731 & 0.887 & 0.802 & 0.972 & $C i$ \\
\hline$P$ & n-Propyl cinnamate & 208.132 & 184.753 & 0.031 & 0.011 & 1.276 & 0.036 & 2.774 & HMDB37700 & 0.754 & 0.628 & 0.881 & $\mathrm{ci}$ \\
\hline$P$ & $\begin{array}{l}\text { Monomethyl glutaric } \\
\text { acid }\end{array}$ & 210.075 & 378.350 & 0.011 & 0.045 & 2.768 & 0.000 & 0.233 & HMDB00858 & 0.913 & 0.835 & 0.991 & $F_{c}$ \\
\hline$P$ & Val-Thr & 219.133 & 365.510 & 0.021 & 0.065 & 2.702 & 0.000 & 0.328 & HMDB29137 & 0.926 & 0.848 & 1.000 & ar \\
\hline $\mathrm{P}$ & Succinic semialdehyde & 222.096 & 252.122 & 0.044 & 0.030 & 1.402 & 0.001 & 1.457 & HMDB01259 & 0.770 & 0.648 & 0.892 & $F_{c}$ \\
\hline$P$ & Phenylpropionylglycine & 230.076 & 385.716 & 0.046 & 0.064 & 2.206 & 0.000 & 0.718 & HMDB00860 & 0.820 & 0.713 & 0.927 & Ci \\
\hline$P$ & 3-Hydroxykynurenine & 242.113 & 374.104 & 0.011 & 0.017 & 2.092 & 0.001 & 0.656 & HMDB00732 & 0.754 & 0.633 & 0.876 & $\mathrm{Ci}$ \\
\hline$P$ & Glu-Thr & 249.108 & 407.789 & 0.188 & 0.298 & 2.002 & 0.000 & 0.631 & HMDB28829 & 0.847 & 0.742 & 0.951 & ar \\
\hline$P$ & N-Acetyl-L-alanine & 263.123 & 406.031 & 0.076 & 0.173 & 2.505 & 0.000 & 0.442 & HMDB00766 & 0.914 & 0.846 & 0.983 & $\mathrm{Ci}$ \\
\hline
\end{tabular}




\begin{tabular}{|c|c|c|c|c|c|c|c|c|c|c|c|c|c|}
\hline$P$ & cis-9-Palmitoleic acid & 296.258 & 36.398 & 0.157 & 0.650 & 1.699 & 0.007 & 0.241 & HMDB03229 & 0.831 & 0.724 & 0.938 & $\mathrm{~F}$ \\
\hline$P$ & $\begin{array}{l}\text { 16-Hydroxypalmitic } \\
\text { acid }\end{array}$ & 314.268 & 39.758 & 0.017 & 0.050 & 1.531 & 0.004 & 0.350 & HMDB06294 & 0.801 & 0.686 & 0.916 & $F_{c}$ \\
\hline$P$ & Phe-Trp & 352.164 & 158.440 & 0.020 & 0.015 & 1.436 & 0.000 & 1.285 & HMDB29006 & 0.801 & 0.686 & 0.916 & $\begin{array}{l}\text { ar } \\
\text { ar }\end{array}$ \\
\hline$P$ & $\begin{array}{l}\text { D-erythro-Sphingosine- } \\
\text { 1-phosphate }\end{array}$ & 380.255 & 262.658 & 0.030 & 0.039 & 1.834 & 0.000 & 0.775 & HMDB00277 & 0.830 & 0.721 & 0.939 & $\mathrm{pr}$ \\
\hline$P$ & O-Desmethylnaproxen & 433.156 & 379.680 & 0.009 & 0.027 & 2.577 & 0.000 & 0.347 & HMDB13989 & 0.866 & 0.778 & 0.953 & $\mathrm{Di}$ \\
\hline
\end{tabular}

\section{Figures}

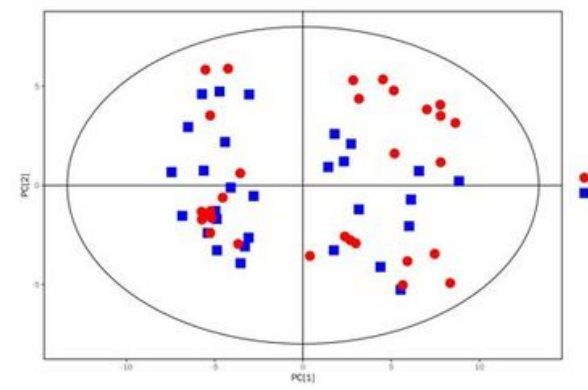

a. Score scatter plot for PCA model (positive ion model)

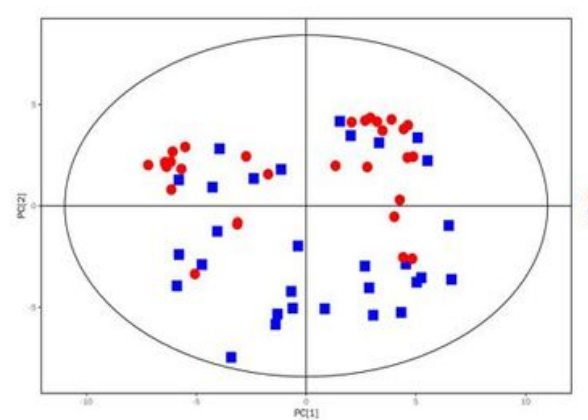

b. Score scatter plot for PCA model (negative ion model )

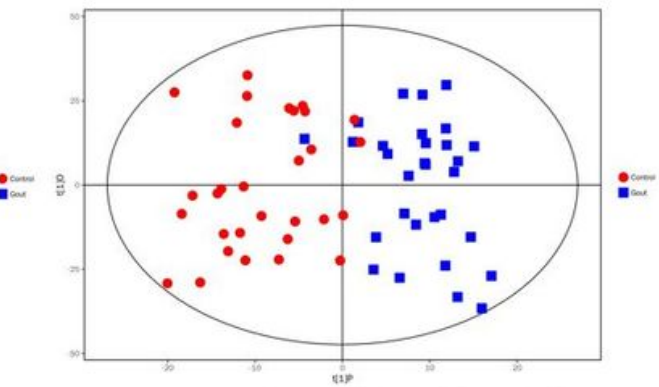

c. Score scatter plot of OPLS-DA model (positive ion model)

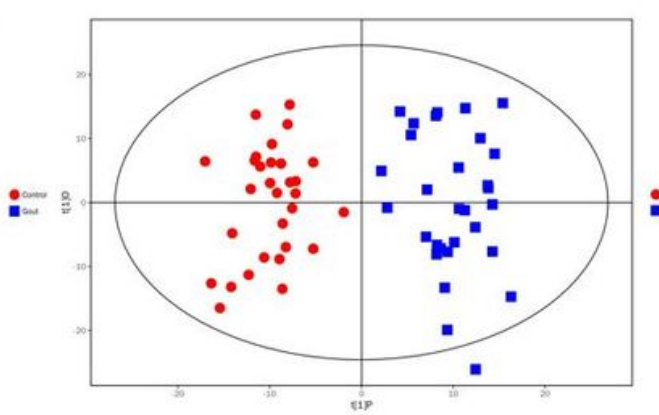

d. Score scatter plot of OPLS-DA model f. Permutation test of OPLS-DA (negative ion model)

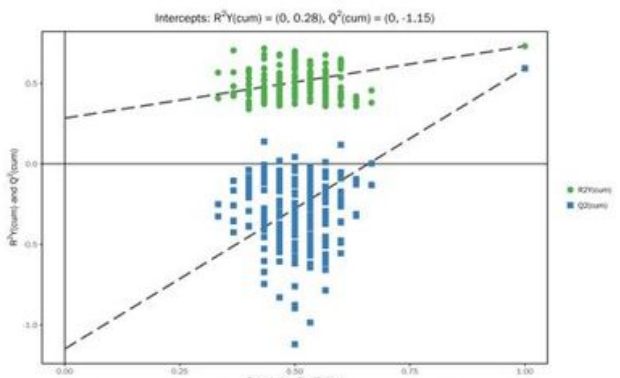

e. Permutation test of OPLS-DA model (positive ion model)

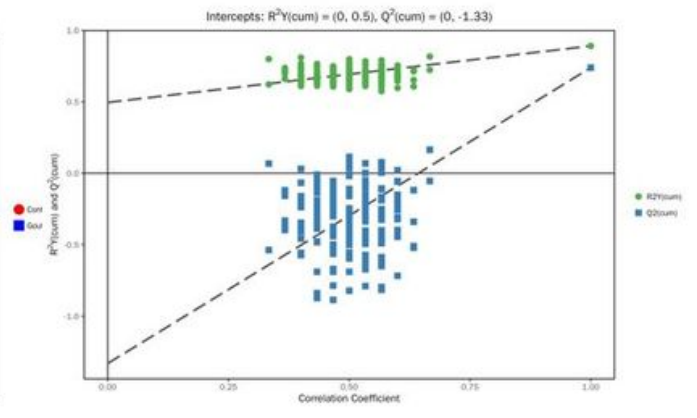

model (negative ion model)

Figure 1

Score scatter plot for PCA and OPLS-DA for group Gout vs Healthy Control. 


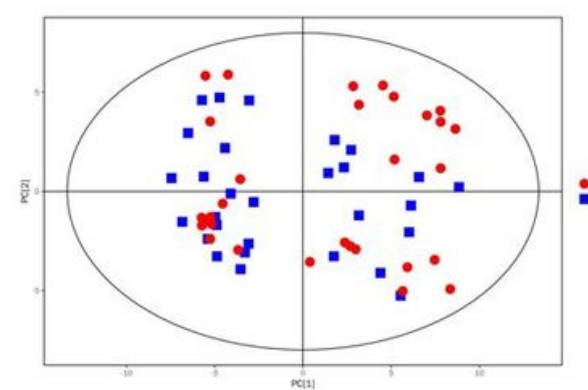

a. Score scatter plot for PCA model (positive ion model)

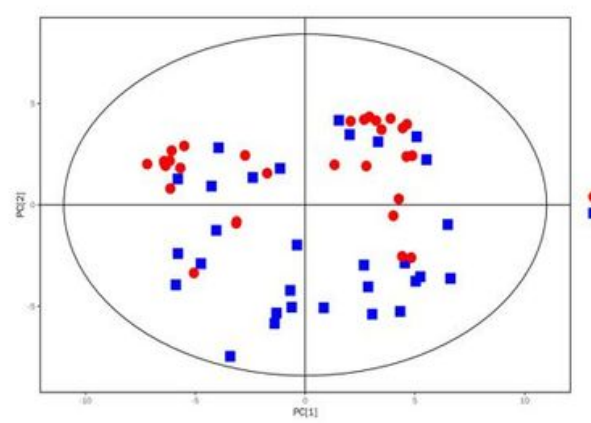

b. Score scatter plot for PCA model (negative ion model )

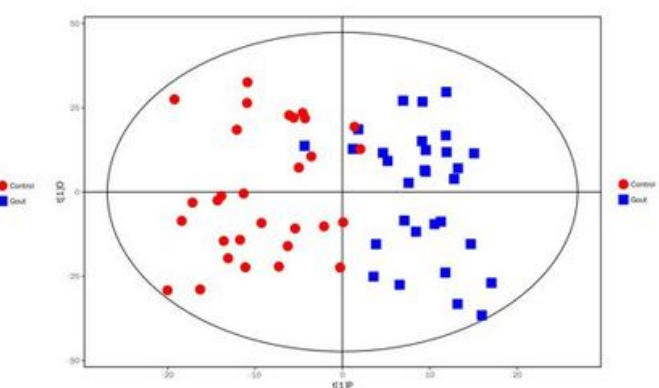

c. Score scatter plot of OPLS-DA model (positive ion model)

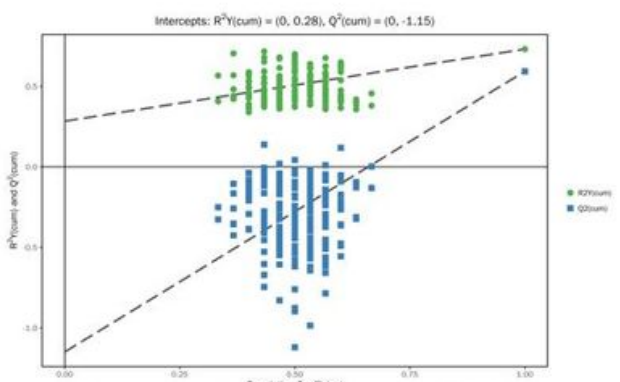

e. Permutation test of OPLS-DA model (positive ion model)

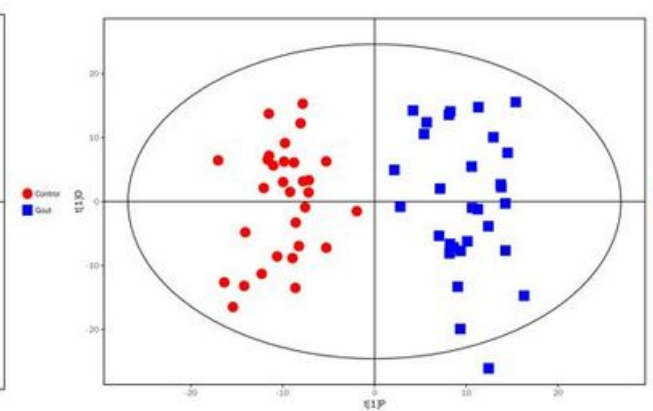

d. Score scatter plot of OPLS-DA model (negative ion model)

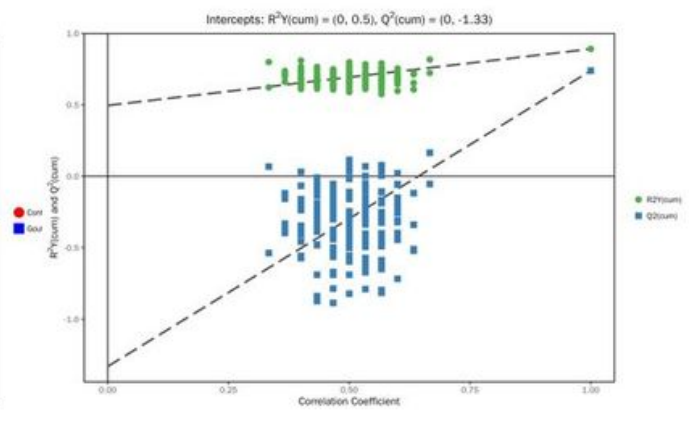

model (negative ion model )

Figure 1

Score scatter plot for PCA and OPLS-DA for group Gout vs Healthy Control.

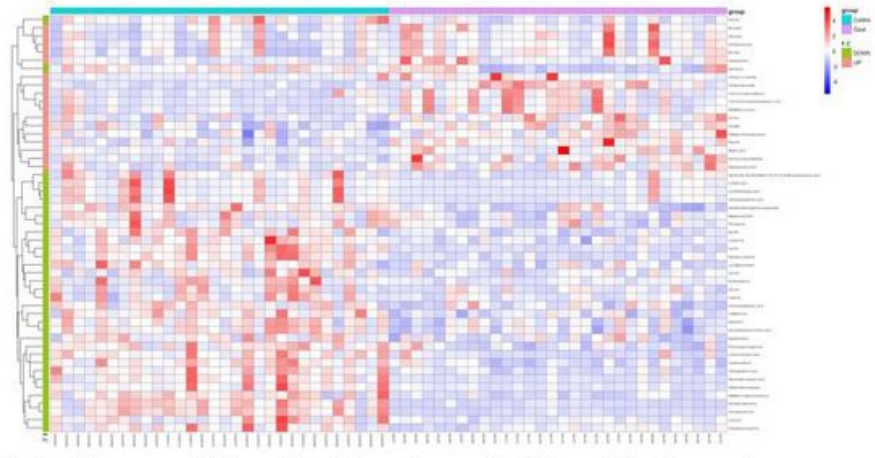

Fig 2a. Heatmap of hierarchical clustering analysis in positive ion mode

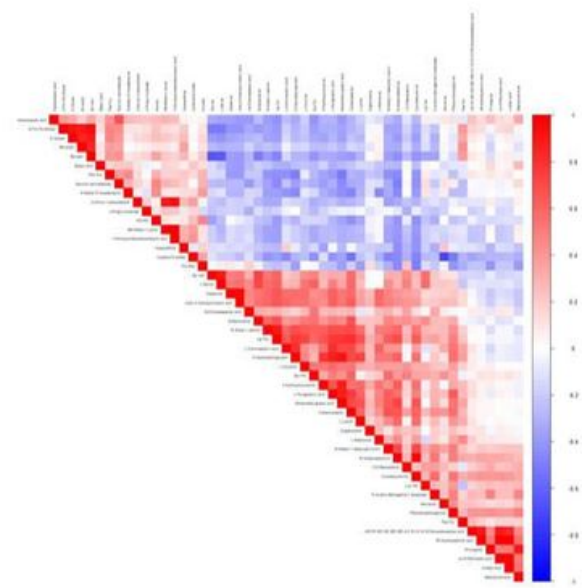

Fig 2c. Heatmap of correlation analysis in positive ion mode

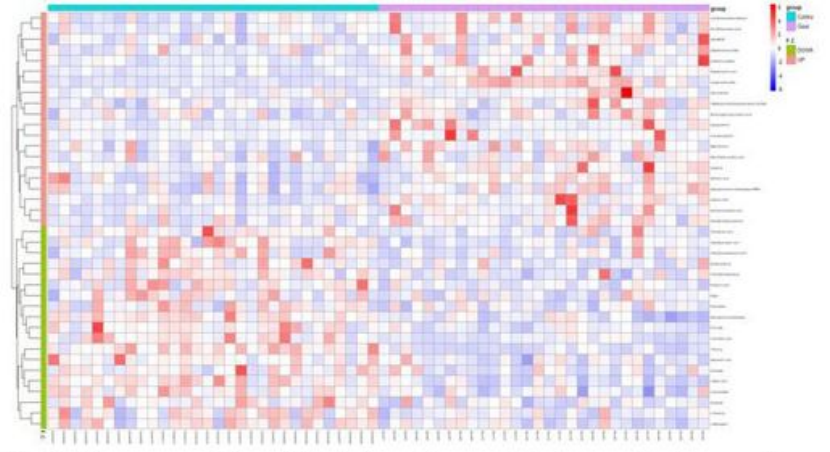

Fig 2 b. Heatmap of hierarchical clustering analysis in negative ion mode

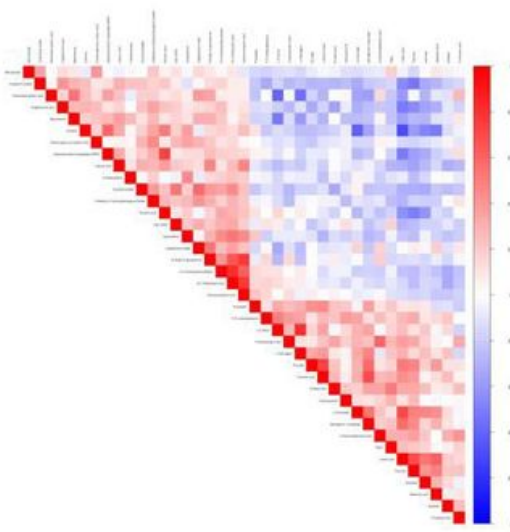

Fig 2 d. Heatmap of correlation analysis in negative ion mode 
Heatmap analysis.

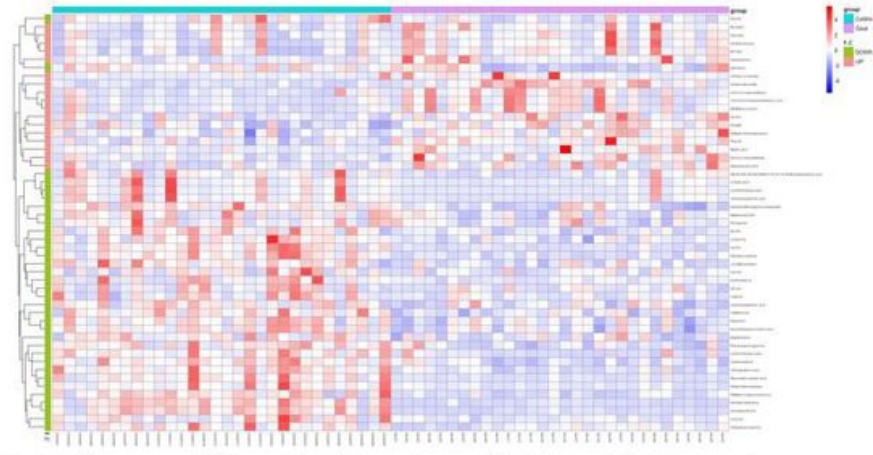

Fig 2a. Heatmap of hierarchical clustering analysis in positive ion mode

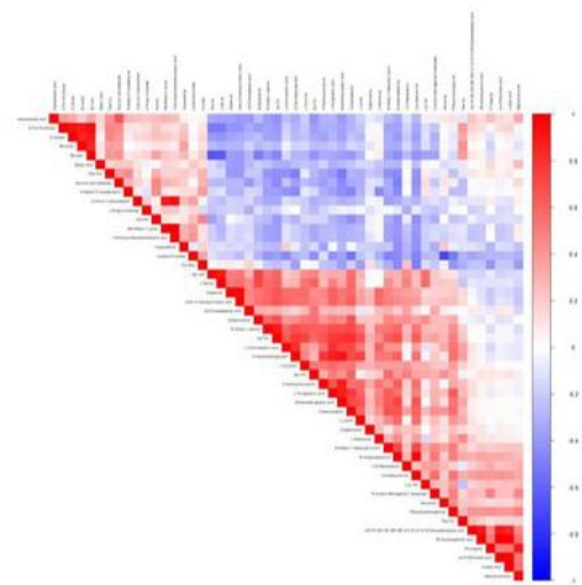

Fig 2c. Heatmap of correlation analysis in positive ion mode

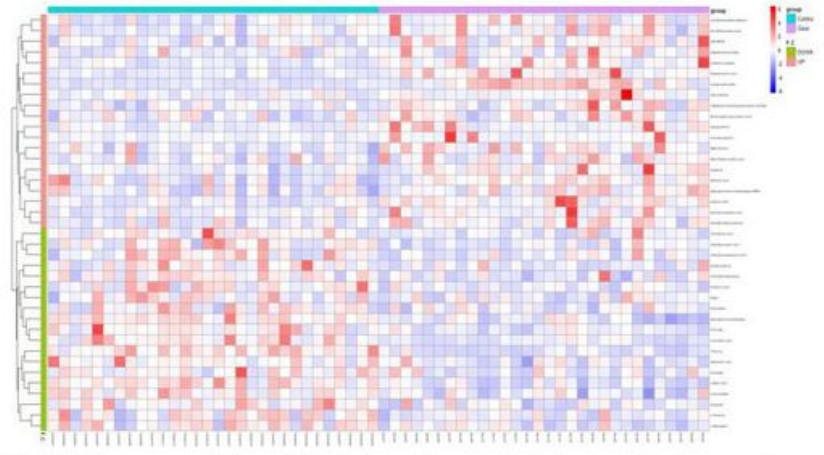

Fig 2 b. Heatmap of hierarchical clustering analysis in negative ion mode

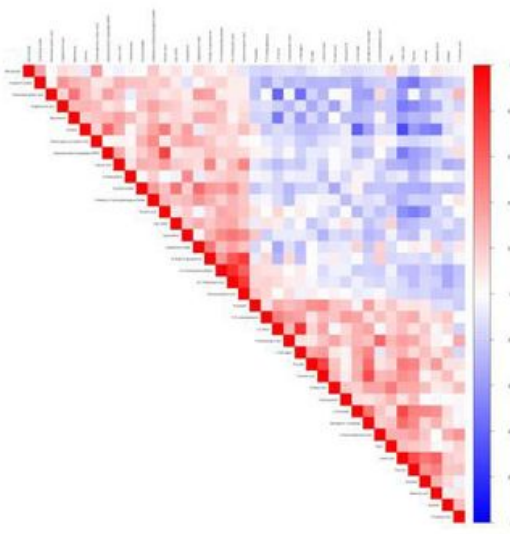

Fig 2d. Heatmap of correlation analysis in negative ion mode

Figure 2

Heatmap analysis.

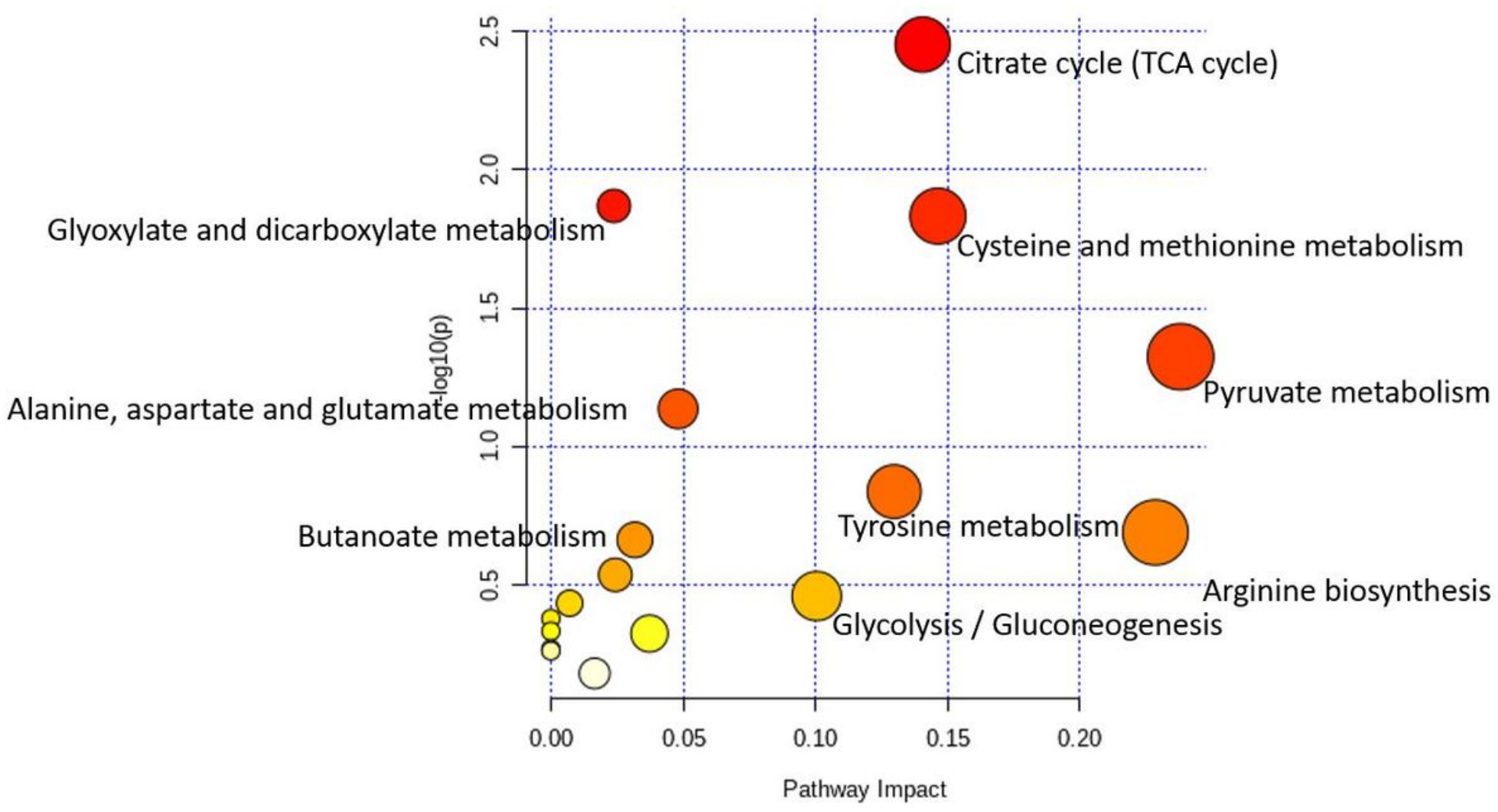


Pathway analysis for group Gout vs Healthy Control.

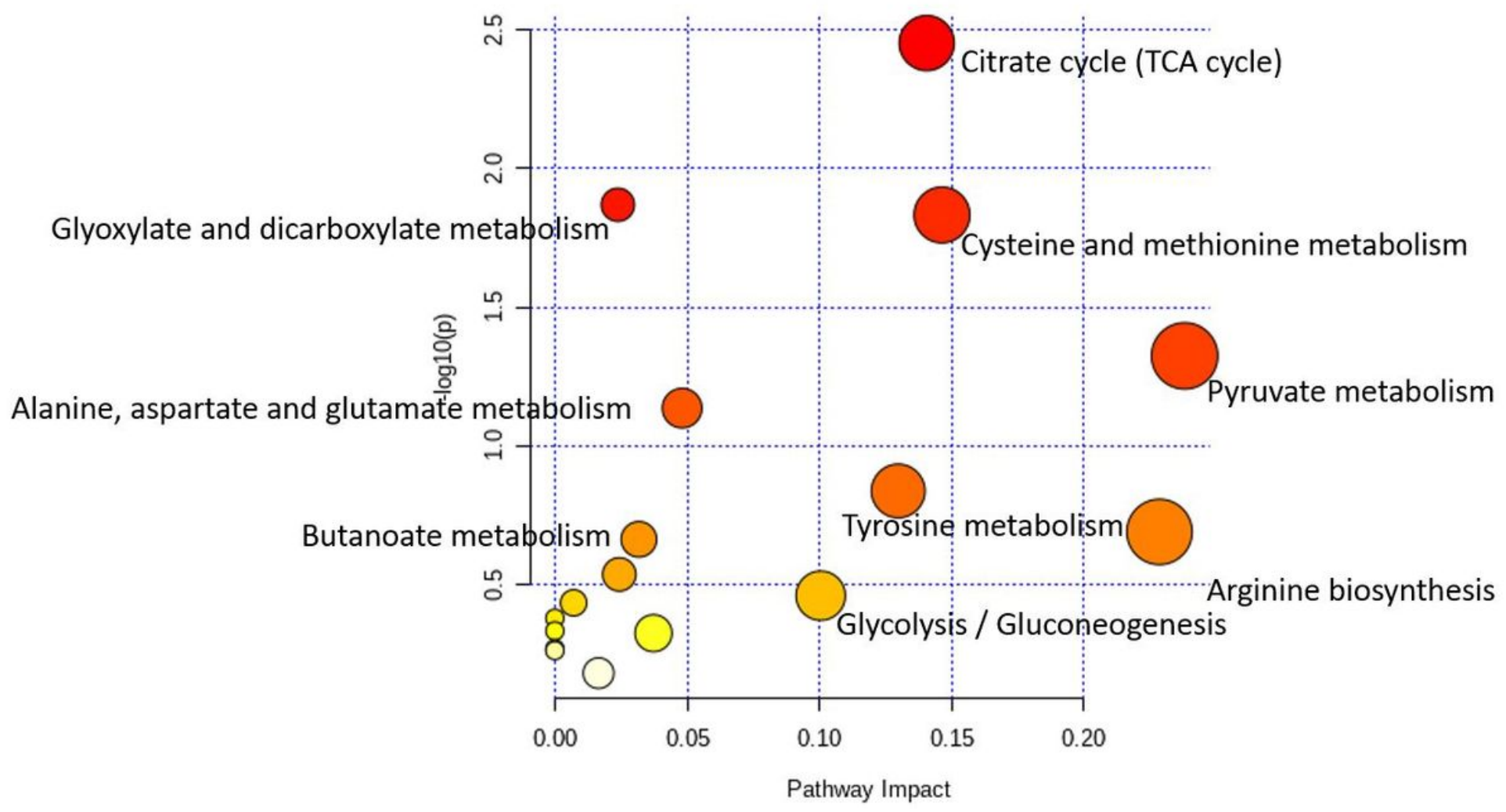

Figure 3

Pathway analysis for group Gout vs Healthy Control. 


\section{Metabolite Sets Enrichment Overview}

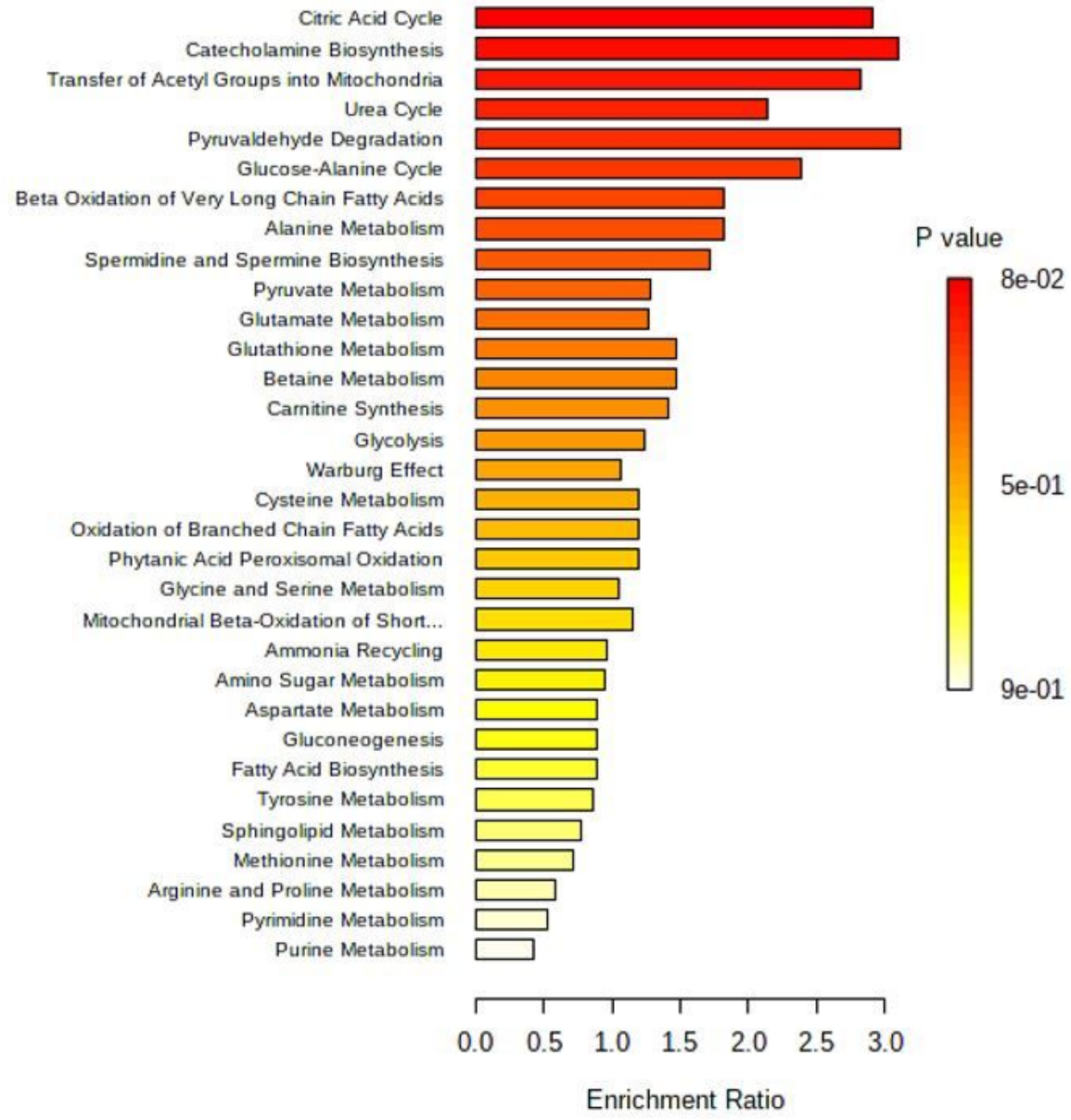

Figure 4

Enrichment pathway analysis for group Gout vs Healthy Control. 


\section{Metabolite Sets Enrichment Overview}

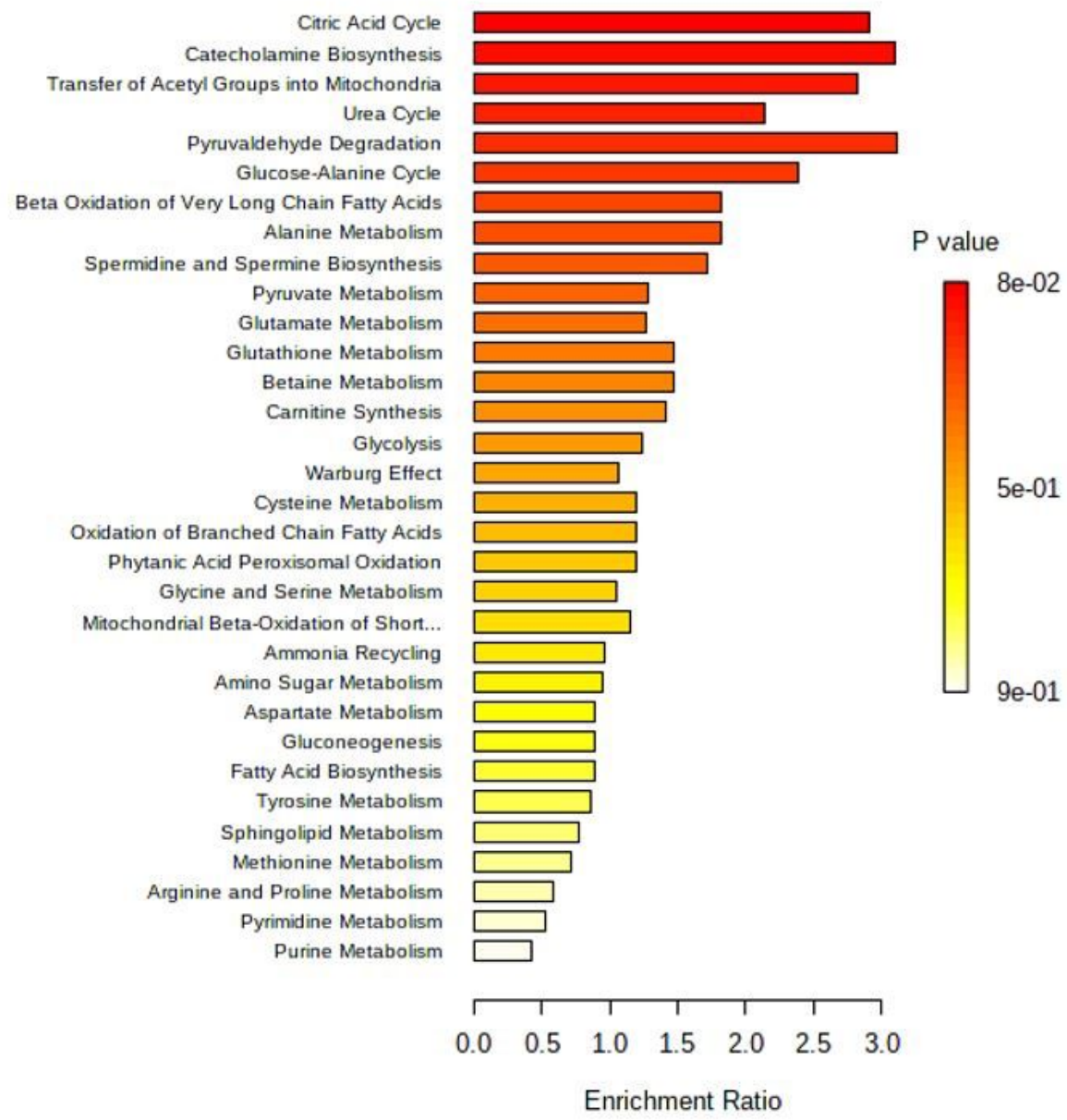

Figure 4

Enrichment pathway analysis for group Gout vs Healthy Control.

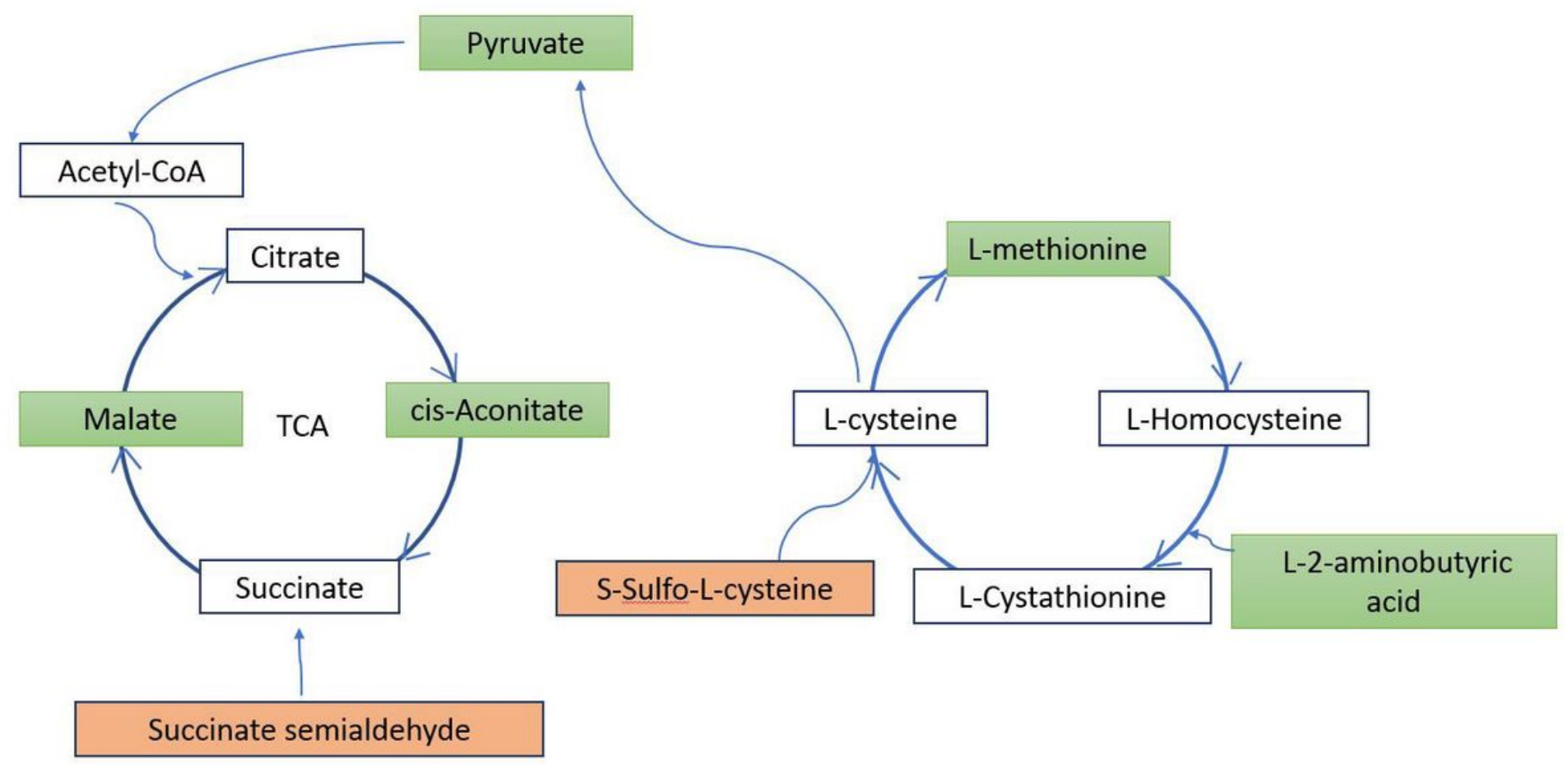


Figure 5

Schematic representation of disturbed metabolic pathways. Metabolites marked in organge, green and white indicate metabolites that significantly increased, decreased or remain unchanged or were not measured, respectively.

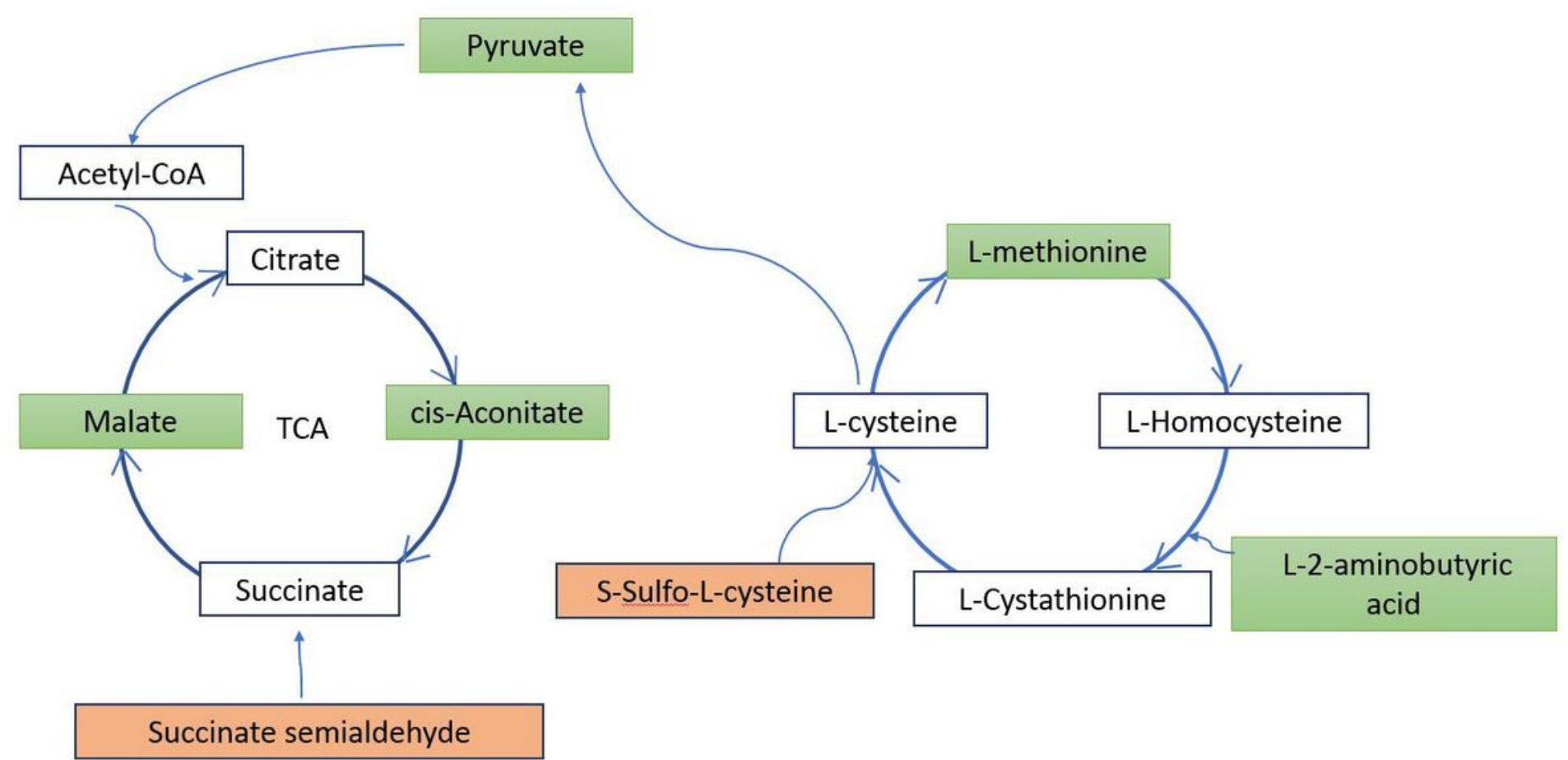

Figure 5

Schematic representation of disturbed metabolic pathways. Metabolites marked in organge, green and white indicate metabolites that significantly increased, decreased or remain unchanged or were not measured, respectively. 


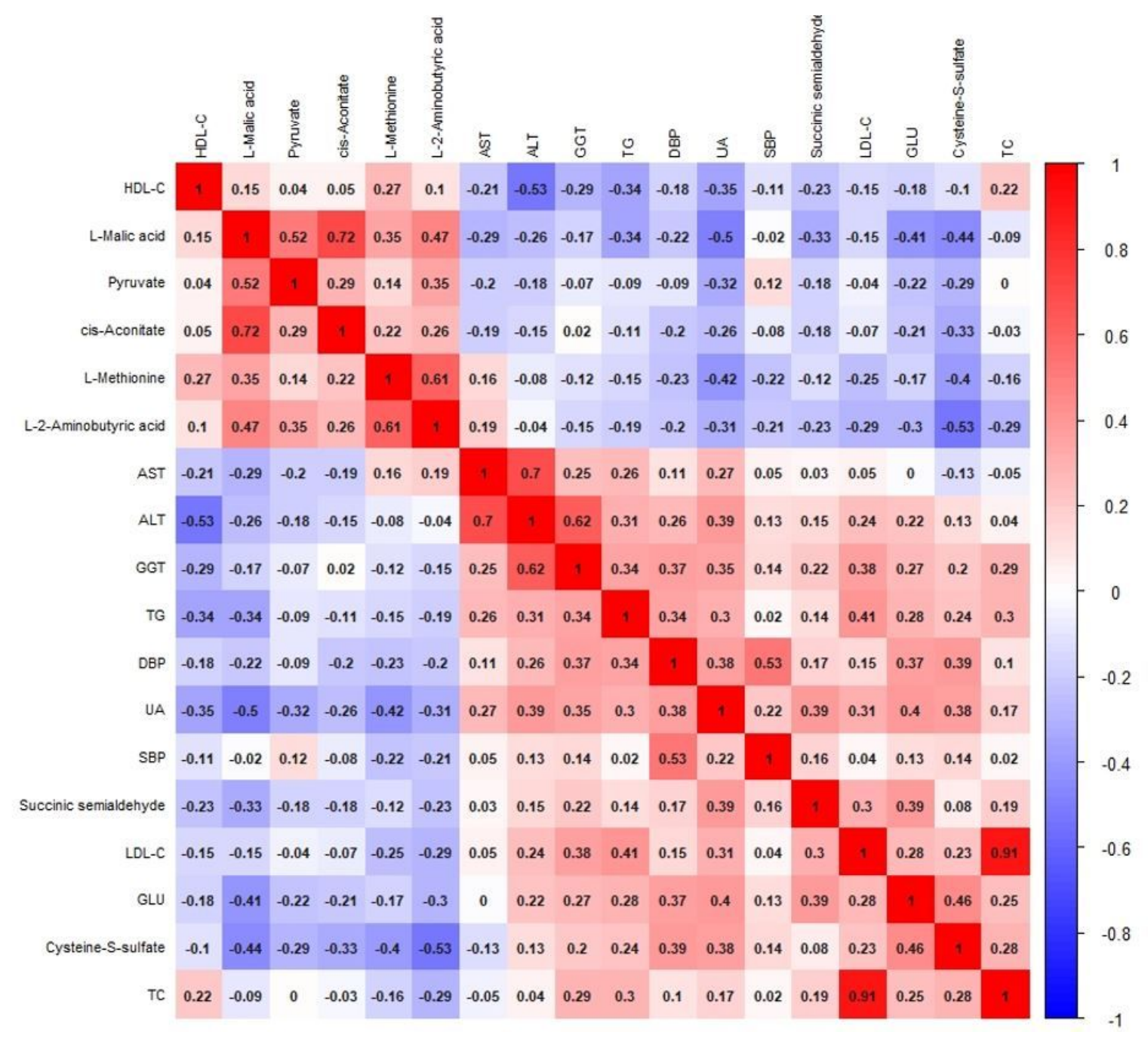

\section{Figure 6}

Correlation analysis of clinical features and metabolites. 


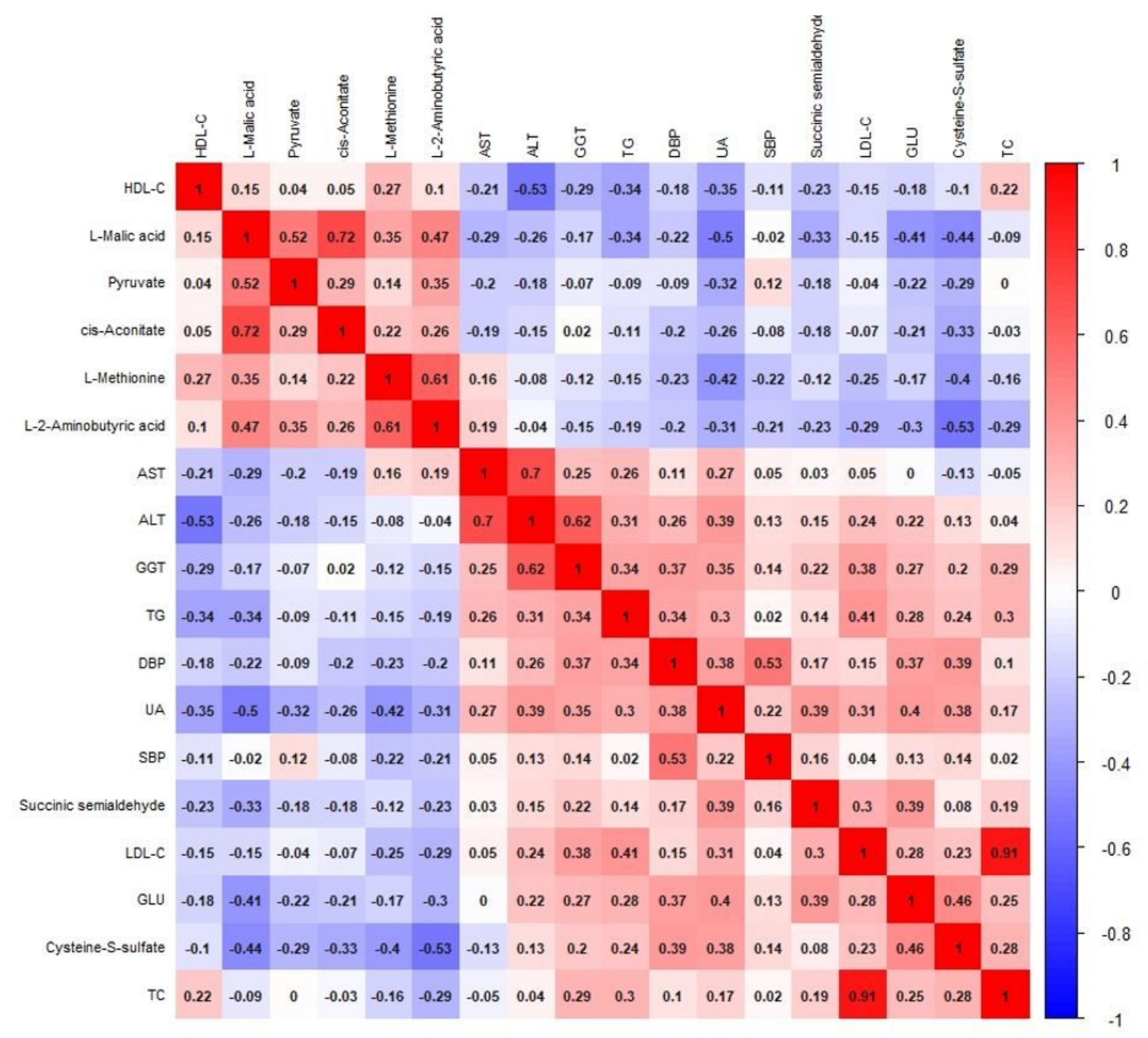

\section{Figure 6}

Correlation analysis of clinical features and metabolites. 